\title{
Studies on Pyrazolones
}

\author{
X. Reactions of Pyrazole Blue with Cyanoacetamide and \\ Malononitrile
}

G UNNEL WESTÖ̈̈

Institute of Organic Chemistry, University of Lund, Lund, Sweden

\begin{abstract}
Cyanoacetamide and malononitrile add to pyrazole blue by normal Michael additions with formation of compounds V and VII. The malononitrile derivative (VII) rearranges readily to the cyclic compound VI.
\end{abstract}

In

a previous paper ${ }^{1}$ it was shown that ethyl cyanoacetate reacts with pyrazole blue (I) with the formation of two different adducts (II and III). Both adducts were readily hydrolysed by alkali with the formation of IV.

In the present investigation, one adduct only, a monobasic acid for which the structure $V$ is proposed, was obtained from the reaction of pyrazole blue with cyanoacetamide. Compound V showed no tendency to ring closure and in this behaviour resembled IV and contrasted with II. The UV absorption curve of V (Fig. 1) showed a striking resemblance to the corresponding curves of II and IV.

Addition of malononitrile to pyrazole blue in the presence of a small amount of piperidine furnished mainly a product, which was obviously the iminolactone VI (a, b, c), since it had a neutral reaction and a UV absorption very similar to that of III ${ }^{1}$. In the absence of a catalyst, a mixture of VI and a dibasic acid was obtained. The same dibasic acid was formed on treatment of VI with excess of dilute alkali at room temperature. It had a UV absorption curve quite analogous to the corresponding curves of II, IV and V (Fig. 1). Hence structure VII is proposed for this compound.

As in the case of II, VII could be easily transformed into the corresponding cyclization product. Thus a solution of VII in ethanol was slowly isomerized to form VI. The transformation was rapid when a small amount of piperidine was added. Dry hydrogen chloride reacted with VII in ether solution with the formation of a hydrochloride of VI.

The IR spectrum of $V$ showed a distinct $\mathrm{C} \equiv \mathrm{N}$ stretching band at $2240 \pm 15$ $\mathrm{cm}^{-1}$, whereas in VII the nitrile band was very weak in spite of the two $\mathrm{C} \equiv \mathbf{N}$

Acta Chem. Scand. 10 (1956) No. 4 


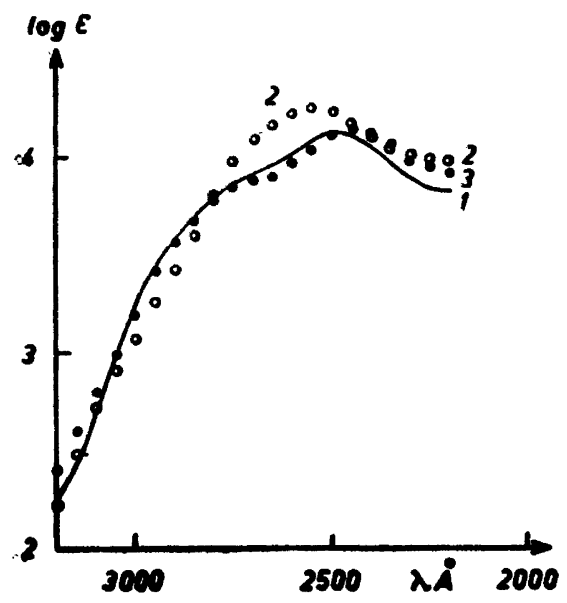

Fig. 1. UV absorption curves of compound $V$ (1), VI (2) and VII (3) in ethanol solutions.

groups present in the molecule. A similar weakening of a $\mathbf{C} \equiv \mathbf{N}$ band was described in 1952 by Kitson and Griffith ${ }^{2}$, particularly in molecules with oxygen-containing groups. With compound IV the same effect was observed ${ }^{2}$.

The spectrum of VI exhibited an intense $\mathrm{C} \equiv \mathrm{N}$ absorption. The band was, however, situated at $2190 \pm 15 \mathrm{~cm}^{-1}$. This indicates that the $\mathrm{C} \equiv \mathbf{N}$ group is in conjugation and hence that the structure of VI is essentially that of VI b. According to Kitson and Griffith the IR absorption of a nitrile group in conjugation with an olefinic double bond is found at $2225 \pm 8 \mathrm{~cm}^{-1}$. Thus the extension of the resonance to the free electron pairs of the oxygen and nitrogen atoms in the $\beta$-position of the cyano group in VI b gives rise to a marked shift of the nitrile band towards longer wavelengths.

The acidic strength of the hydrogen atoms in the $\alpha$-position of the cyano groups in II, IV, V and VII is rather different. In ethanol-water solution using alizarine yellow indicator the a-hydrogen atom is neutral in compounds IV and V. In II it can be titrated with barium hydroxide solution (alizarine yellow indicator), though with a very poor end point. It is possible, however, to titrate compound VII with a sharp end point using thymolphthalein indicator. Thus in II and VII the acidifying effect of the bispyrazolonyl group, which is electron attracting and hence stabilizes the carbanion, is striking, for ethyl cyanoacetate exhibits very weakly acidic properties under the above conditions, and the ionization constant of malononitrile is $6.5 \times 10^{-12}$ according to Pearson and Dillon ${ }^{3}$.

In dimethylformamide solution, using azo violet indicator, the effect of the bispyrazolonyl group is not noticeable on titration with sodium methoxide 4 . Both malononitrile, ethyl cyanoacetate and their bispyrazolonyl derivatives are accurately titrated. Cyanoacetamide and compound $V$ can be titrated, though with a very poor end point. In cyanoacetic acid as in compound IV the $\alpha$-hydrogen has no acidic properties on titration.

Thus compounds II and VII differ from IV and V both in their greater tendency to ring closure and in the greater acidity of the hydrogen atoms in the $\alpha$-position of the nitrile groups. One reason for these properties is the grea- 


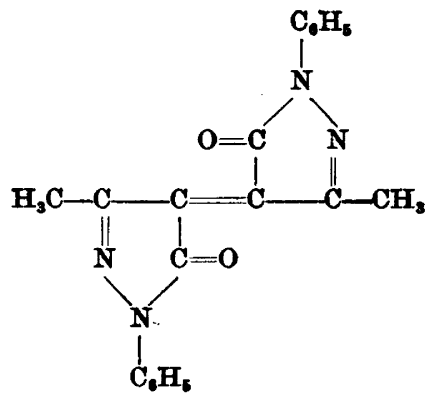

I

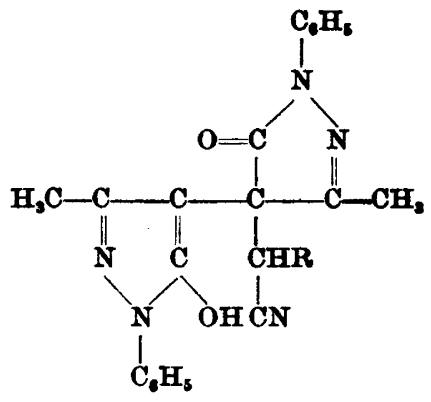

II $R=\mathrm{COOC}_{2} \mathrm{H}_{1}$

IV $R=$ COOH

V $\mathbf{R}=\mathrm{CONH}$,

VII $\mathbf{R}=\mathbf{C N}$

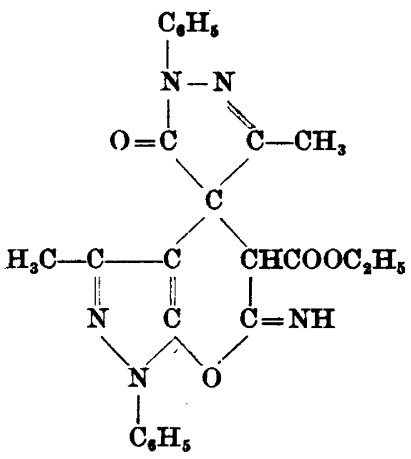

III a

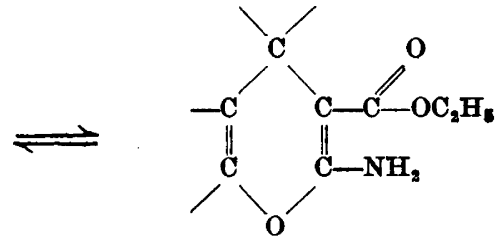

III b

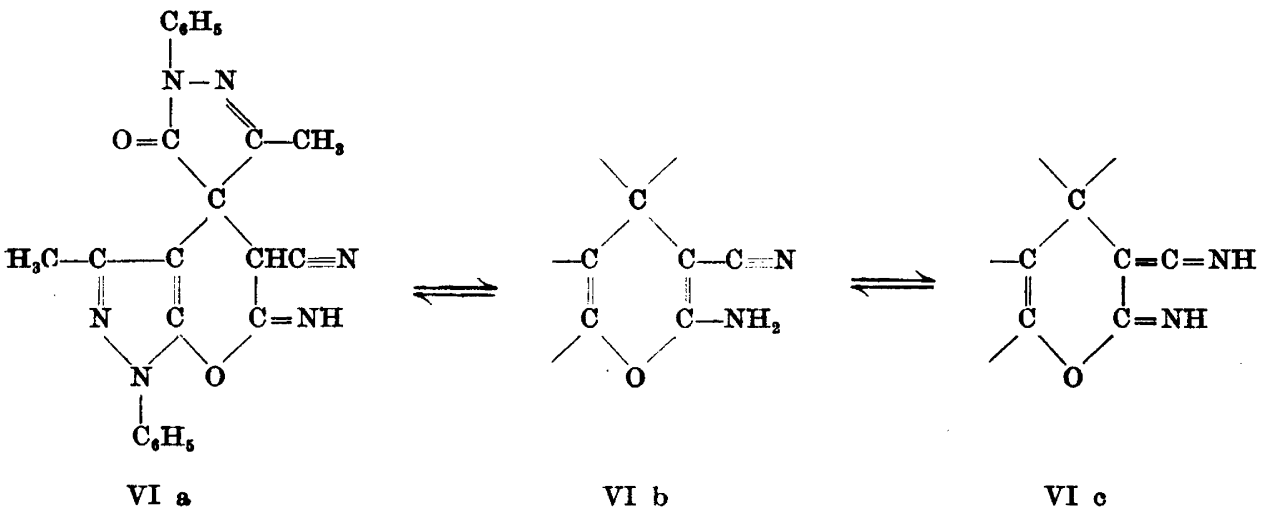

Acta Chem. Scand. 10 (1956) No. 4 
ter resonance interaction with conjugated groups or free electron pairs of the carbethoxy group of II and still more of the cyano group of VII, compared to the carboxyl and amide groups of IV and V. The resonance causes stabilizstion of the ring compounds III $b$ and VI $b$. It also stabilizes the anions of II and VII.

\section{EXPERIMENTAL}

Preparation of compound $V$ from pyrazole blue and cyanoacetamide. A mixture of cyanoacetamide $(4.0 \mathrm{~g})$, pyrazole blue $(16.2 \mathrm{~g})$, chloroform $(70 \mathrm{ml})$, ethanol $(10 \mathrm{ml})$ and piperidine $(0.4 \mathrm{ml})$ was shaken overnight. The colourless precipitate formed was filtered off and washed with ethanol. Yield of air-dried product: $20.0 \mathrm{~g}$. It was purified by crystallization from ethanol. The compound adsorbed the solvent strongly. It was dried at $0.1 \mathrm{~mm} \mathrm{Hg}, 78^{\circ}$, over phosphoric anhydride and was afterwards hygroscopic. (Found: C 64.0; $\mathrm{H} 4.6$; $\mathrm{N}$ 19.4; equiv. wt. 430. $\mathrm{C}_{23} \mathrm{H}_{20} \mathrm{~N}_{8} \mathrm{O}_{3}$ requires $\mathrm{C} 64.5 ; \mathrm{H} 4.7 ; \mathrm{N} 19.6$; equiv. wt. 428.5.) For UV absorption curve, see Fig. 1.

Preparation of VI from pyrazole blue and malononitrile. Malononitrile (3.15 g), pyrazole blue $(15.6 \mathrm{~g})$, chloroform $(90 \mathrm{ml})$ and piperidine $(0.3 \mathrm{ml})$ were mixed together. Decolorization took place in a few minutes, and crystals of VI separated. After cooling with iced water, the precipitate was filtered off and washed with cold ethanol. Yield: $17.2 \mathrm{~g}$ of air-dried product. It was purified by recrystallization from ethanol and dried in vacuo $(0.1 \mathrm{~mm} \mathrm{Hg})$ at $100^{\circ}, \mathrm{m} . \mathrm{p}$. about $240^{\circ}$ (decomp.). The product was rapidly isomerized into VII even by $0.1 \mathrm{~N}$ alkali solution. (Found: $\mathrm{C} 67.1 ; \mathrm{H} 4.5 ; \mathrm{N} 20.5 . \mathrm{C}_{23} \mathrm{H}_{10} \mathrm{~N}_{6} \mathrm{O}$, requires C 67.3; H 4.4; N 20.5.) UV absorption curve see Fig. 1.

Transformation of VI into VII by addition of alkali. A mixture of compound VI $(7.0 \mathrm{~g})$, ethanol $(35 \mathrm{ml})$ and $2.5 \mathrm{~N}$ sodium hydroxide solution $(35 \mathrm{ml})$ was kept overnight and then poured into $\mathrm{N}$ hydrochloric acid. The product which separated $(6.9 \mathrm{~g})$ was collected by filtration, washed with water and air-dried. After extraction of impurities with ethyl acetate it was dried under reduced pressure $(0.1 \mathrm{~mm} \mathrm{Hg})$ at $78^{\circ}$ over phosphoric anhydride. The product melted at $230-240^{\circ}$ (decomp.). (Found: $C 67.3$; H 4.5; $\mathbf{N} 20.4$; equiv. wt. 203. $\mathrm{C}_{23} \mathrm{H}_{28} \mathrm{~N}_{6} \mathrm{O}_{2}$ requires $\mathrm{C} 67.3 ; \mathrm{H} \mathrm{4.4;} \mathrm{N} 20.5$; equiv. wt. 205.) The UV absorption curve is seen in Fig. 1.

Transformation of VII into VI using piperidine as a catalyst. A solution of compound VII $(0.85 \mathrm{~g})$ and two drops of piperidine in ethanol $(25 \mathrm{ml})$ was kept overnight at room temperature. The product formed was precipitated by addition of $\mathrm{N}$ hydrochloric acid and water, filtered and washed with water. It was purified by crystallization from ethanol. The neutral product obtained was easily hydrolysed by alkali in a similar manner to compound VI. UV absorption curve and analyses were identical with the data of VI.

Transformation of VII into VI by hydrogen chloride. A suspension of VII in ether was treated with dry hydrogen chloride, and the resulting salt was filtered off and washed with ether. (Equiv. wt. of the air-dried product on titration using bromophenolblue indicator: 470. Calc. for $\mathrm{C}_{28} \mathrm{H}_{18} \mathrm{~N}_{6} \mathrm{O}_{2}, \mathrm{HCl}: 447$.) The free base was isolated by precipitation of the ethanol solution of the salt with water. According to analyses, UV absorption curve and melting point, it was identical with compound VI.

The analyses were performed by Miss M. Westerdahl, Department of Analytical Chemistry, University of Lund. Dr. Andreas Rosenberg, University of Uppsala, carried out the IR measurements.

The author wishes to thank Statens naturvetenskapliga forskningsräd for financial support.

\section{REFERENCES}

1. Westöö, G. Acta Chem. Scand. 10 (1956) 9.

2. Kitson, R. E. and Griffith, N. E. Anal. Chem. 24 (1952) 334.

3. Pearson, R. G. and Dillon, R. L. J. Am. Chem. Soc. 75 (1953) 2439.

4. Fritz, J. S. Anal. Chem. 24 (1952) 674.

Received January 14, 1956. 